\title{
Evaluación del tiempo en que Aedes aegypti coloniza y desarrolla su fase acuática en los lavaderos de ropa de los barrios surorientales de Neiva
}

\author{
Myriam L. Benavides ${ }^{1}$, Luis O. Realpe ${ }^{2}$, Paulina Fajardo ${ }^{3}$
}

\begin{abstract}
Resumen
En 1992, el índice de infestación larvaria por Aedes aegypti fue de $22,5 \%$ en las viviendas de los barrios del sur de Neiva. De junio a septiembre de 1993, en los lavaderos de ropa de 33 viviendas, se midió el tiempo de colonización y se permitió el desarrollo larvario del vector hasta la fase de pupa. A. aegypti colonizó los depósitos de agua en un tiempo de dos días y desarrolló en ellos su fase acúatica en siete días, promedios que sumados permiten proponer para Neiva la limpieza de los lavaderos domésticos de ropa cada nueve días, como una medida de control antivectorial. Igualmente, se correlacionaron algunas características de la alberca y de su utilización con el tiempo de colonización y desarrollo de la fase acuática del vector.
\end{abstract}

\section{Summary}

In 1992, in neighbourhoods in the south-east of Neiva, the Aedes aegypti larval infestation index in homes was $22.5 \%$. Laundry tubs (albercas) were tested in 33 homes from June to September 1993. The time taken by the vector to colonise and to develop as far as its pupal stage was measured. A. aegypti took two days to colonise albercas' areas and developed to its aquatic stage in them in seven, averages which therefore suggest that laundry tubs should be cleaned and washed out every nine days on average as a measure of anti-vectorial control. Some of the tubs characteristics and their relationship to colonisation time and the vector's development to aquatic phase were correlated.

Aedes aegypti es un díptero hematófago de hábitos como vector del dengue y de la fiebre amarilla urbana. Es un mosquito de origen africano que, en el hemisferio occidental, presenta estrecha asociación con los humanos, criándose fuera y al interior de la vivienda en depósitos de agua fabricados por el hombre y, secundariamente, en depósitos naturales (1, 2).

Los criaderos permanentes más importantes y de uso obligado en la región son las albercas o depósitos de agua de los lavaderos de ropa y los tanques elevados de almacenamiento de agua para el consumo; son menos frecuentes los depósitos temporales como botellas y frascos abandonados en los patios de las viviendas (3) que, dada la baja pluviosidad de Neiva $-1300 \mathrm{~mm}$ anuales (4)- permanecen la mayor parte del tiempo secos. Se entiende por criadero a todo cuerpo de agua que puede ser colonizado por la hembra de $A$. aegypti para la ovipostura, eclosión, desarrollo de larvas, pupas y emergencia de adultos.

\footnotetext{
${ }^{1}$ Colegio Jesús María Aguirre, Aipe, Huila.

${ }^{2}$ Colegio Cooperativo del Huila, Neiva, Huila.

${ }^{3}$ Facultad de Salud, Universidad Surcolombiana, Neiva, Huila.
} 
El indice aédico larvario informado por la División de Patologías Tropicales (DPT) para las viviendas de los barrios surorientales, en 1992, fue de 22,5\%. Entre mayo de 1992 y enero de 1993, se presentaron en Neiva 100 casos de dengue hemorrágico (5) lo que hizo necesario realizar fumigaciones casa a casa y aplicación de abate en las albercas.

El estudio pretende establecer el tiempo en que $A$. aegypti coloniza y desarrolla su fase acuática en las albercas domésticas de los barrios surorientales de la ciudad de Neiva, para estimar la frecuencia con que deben lavarse estos depósitos de agua, evitando así la emergencia de adultos y la infestación de la vivienda con el vector. Se busca, además, evaluar si algunas características de las albercas afectan la duración de estos procesos.

\section{Metodología}

En el municipio de Neiva, a $472 \mathrm{msnm}$ y temperatura promedio de $27,5^{\circ} \mathrm{C}$, se eligió el área seis al suroriente de la ciudad, con 22 barrios y 6.743 viviendas como población de estudio. Para determinar el tamaño de la muestra, se aplicó un muestreo aleatorio simple. Con base en el tamaño poblacional de 6.743 viviendas, nivel de confiabilidad de 1,96, error máximo admisible de $10 \%$ y tiempo promedio de colonización de $A$. aegypti de 61 horas con una desviación estándar de 17,87 horas obtenido en un estudio previo (6), el tamaño de la muestra calculada fue de 33 viviendas con sus respectivos lavaderos.

Para seleccionar las viviendas en los 22 barrios, se aplicó la técnica de seleccion sistemática, la cual permite escoger un elemento de cada K-elementos obteniéndose la distribución de las viviendas de la muestra en toda la población.

La comuna seis está formada por barrios de estratos socioeconómicos bajo, medio y medioalto. Las viviendas en su mayoría son de una o dos plantas. En el patio posterior, poseen un lavadero de ropa, el cual consta de un depósito de agua llamado alberca, cubierto parcialmente por una plancha de cemento o fregadero. De construcción prefabricada, algunas albercas están recubiertas por enchape de baldosín porcelanizado que cubre internamente piso y paredes; su volumen es variable y algunas se encuentran bajo techo.

Al inicio del estudio, se realizó una cuidadosa inspección para establecer el índice de infestación de las albercas de las viviendas seleccionadas con larvas o pupas de $A$. aegypti. Posteriormente, en cada alberca, se evacuó el agua y se lavó todo el interior con cepillo, agua y jabón, eliminando así huevecillos que pudieran estar adheridos en las paredes y piso, quedando negativas, además, para larvas y pupas del mosquito.

Hecho esto, se cubrieron totalmente sus paredes internas con cartulina; se procedió a llenar la alberca y a darle el uso normal por parte de los integrantes de la familia. Se utilizó cartulina como material de cubrimiento por su fácil manejo; el absorber bien el agua no interfiere con la ovipostura del mosquito y facilita la observación de los huevos; tiene, además, relativa resistencia frente al agua y es de bajo costo.

Para establecer la presencia de huevos, en cada alberca, se realizaron tres observaciones al día a las 6:00 am, 12:00 m y 6:00 pm. De acuerdo con los hábitos de la hembra de $A$. aegypti, los huevos se buscaron con la ayuda de una lupa sobre la cartulina, ligeramente, por encima del nivel del agua. Una vez que la alberca se hizo positiva para la presencia de huevos, se continuaron las observaciones con el mismo horario hasta observar el desarrollo de larvas y, finalmente, de pupas.

La búsqueda de larvas y pupas se realizó recogiendo muestras de agua con un recipiente de $50 \mathrm{~mL}$, preferiblemente de los rincones más oscuros, teniendo la precaución de evitar mover innecesariamente el agua, por cuanto que se sumergen hasta el fondo impidiendo que se les detecte. El recipiente con cada muestra de agua se llevó a un sitio bien iluminado para revisar su contenido, usando la lupa para buscar larvas de primer estadio. Cuando se observó la presencia de 
pupas, en forma inmediata se procedió a evacuar el agua de la alberca para evitar la emergencia de adultos. De junio a septiembre de 1993, se realizaron cinco muestreos de este tipo en cada alberca. Al finalizar el estudio, las albercas se lavaron quedando negativas para huevos, larvas y pupas de $A$. aegypti.

Se llevaron muestras de larvas al Laboratorio de Microbiología de la Facultad de Salud para confirmar su identificación, utilizando la clave de Forattini (6).

Para cada muestreo, se registró el día y la hora de llenado de la alberca, de observación de oviposturas en la cartulina, de las primeras larvas y de las primeras pupas. El estudio se realizó en el segundo período seco del año y para establecer las condiciones climáticas, se midieron las variaciones de temperatura del aire y del agua y la humedad relativa del lugar a la 6:00 am, 12:00 m y 6:00 pm.

Con el propósito de correlacionar algunas características de las albercas con el tiempo de colonización y desarrollo de la fase acuática del mosquito, se registró el volumen, la presencia de enchape y de techo $\mathrm{y}$, mediante entrevista, se estableció el número de horas semanales que el ama de casa dedicaba al lavado de la ropa.

\section{Resultados y discusión}

El promedio de temperatura atmosférica fue de $28,2^{\circ} \mathrm{C}$, la temperatura del agua de $25^{\circ} \mathrm{C}$ y la humedad relativa de $60 \%$; al inicio del estudio, $84,8 \%$ de las albercas estaban positivas para larvas o pupas de $A$. aegypti. El $15,2 \%$ fueron negativas y en ellas se encontraron pequeñas bolsas de tela que contenían el larvicida abate, aplicado por la División de Patologías Tropicales.

\section{Tiempo de colonización}

Corresponde al tiempo en que la hembra del vector reconoce la alberca como nicho ecológico y se observa sobre la cartulina la presencia de sus huevos. A. aegypti colonizó $30,3 \%$ de las albercas en un tiempo que está entre 2,1 y 2,4 días y, en $85 \%$ de las albercas, aproximadamente, el tiempo de colonización es menor o igual a 2,4 días (cuadro 1).

\section{Duración de la fase de larva}

Debido a la dificultad para observar las larvas de primer estadio, se consideró que la duración de la fase de Jarva incluía el tiempo de incubación del huevo y el tiempo de la etapa larval hasta observar las primeras pupas. En $30,3 \%$ de las albercas, la duración de la fase de larva está entre 6,6 y 7,4 días y en $66,7 \%$ es menor o igual a 7,4 días (cuadro 2).

El promedio del tiempo total medido de la fase acúatica de $A$. aegypti es de 9 días, que puede ser tan corto como 6,1 y tan largo como 12,1 días (cuadro 3). Este tiempo corresponde a la frecuencia con que el ama de casa debe limpiar la alberca doméstica para evitar la emergencia de adultos.

\section{Características de las albercas}

En general, las albercas están ubicadas en el patio, unidas a la pared que las separa de la vivienda. Las de construcción prefabricada presentan notoria humedad en sus paredes. El volumen promedio es de $1 \mathrm{~m}^{3} ; 66,7 \%$ carece de enchape y se encuentra bajo techo. Su uso promedio está entre 12 y 15 horas semanales.

Para establecer el grado de influencia de las características observadas en las albercas

Cuadro 1. Colonización de A. aegypti en tanques de lavaderos.

\begin{tabular}{|c|c|c|c|}
\hline $\begin{array}{l}\text { Tiempo } \\
\text { (días) }\end{array}$ & $\begin{array}{c}\text { Frecuencia } \\
n\end{array}$ & $\begin{array}{c}\text { Tanques } \\
\%\end{array}$ & $\begin{array}{c}\text { Acumulado de tanques } \\
\%\end{array}$ \\
\hline $1,2-1,5$ & 3 & 9,1 & 9,1 \\
\hline $1,5-1,8$ & 7 & 21,2 & 30,3 \\
\hline $1,8-2,1$ & 8 & 24,3 & 54,6 \\
\hline $2,1-2,4$ & 10 & 30,3 & 84,9 \\
\hline $2,4-2,7$ & 4 & 12,1 & 97,0 \\
\hline $2,7-3,0$ & 1 & 3,0 & 100,0 \\
\hline Total & 33 & & \\
\hline
\end{tabular}


Cuadro 2. Desarrollo de larvas de A. aegypti en tanques de lavaderos.

\begin{tabular}{cccc}
\hline $\begin{array}{l}\text { Tiempo } \\
\text { (días) }\end{array}$ & Frecuencia & $\begin{array}{c}\text { Tanques } \\
\%\end{array}$ & $\begin{array}{c}\text { Acumulado de tanques } \\
\%\end{array}$ \\
\hline $4,9-5,7$ & 4 & 12,1 & 12,1 \\
$5,7-6,6$ & 8 & 24,3 & 35,4 \\
$6,5-7,4$ & 10 & 30,3 & 66,7 \\
$7,4-8,2$ & 9 & 27,3 & 99,0 \\
$8,2-9,1$ & 2 & 6,0 & 100,0 \\
Total & $\mathbf{3 3}$ & & \\
\hline
\end{tabular}

Cuadro 3. Colonizacion y fase acuática de $A$. aegypti en tanques de lavaderos.

\begin{tabular}{lccc}
\hline \multirow{2}{*}{$\begin{array}{c}\text { Etapas de la } \\
\text { fase acuática }\end{array}$} & Promedio & Mínimo & Máximo \\
\cline { 2 - 4 } & 2,0 & 1,2 & 3,0 \\
Colonización & 7,0 & 4,9 & 9,1 \\
Fase de larva & 9,0 & 6,1 & 12,1 \\
Tiempo total medido & & Tías \\
\hline
\end{tabular}

domésticas sobre la duración de las etapas del ciclo acuático de $A$. aegypti, se realizó un análisis de regresión y correlación, el cual indica que no hay asociación lineal entre el tiempo de colonización y el tiempo de duración de la fase de larva con las características de volumen, presencia de enchape, presencia de techo y del uso semanal de las albercas; luego, estas características no parecen influir en el tiempo de colonización ni en la duración de la fase de larva de $A$. aegypti, probablemente por los altos índices de infestación del área y porque las condiciones de temperatura ambiental y temperatura del agua del criadero son óptimas para el desarrollo larvario.

\section{Referencias}

1. Mattingly PF. Genetical aspects of the Aedes aegypti problem. I. Toxonomy and bionomics. Ann Trop Med Parasitol 1957;51(4):392-408.
2. Tinker ME, Olano VA. Ecología del Aedes aegypti en un pueblo de Colombia, Suramérica. Biomédica 1993;13(1):5-14.

3. Cedeño MC. Neiva al filo del milenio: historia de conquista, colonización e identidad. Santa Fe de Bogotá: Kimpres 1993:375.

4. Lozano G. El dengue hemorrágico en el Huila (mayo 1992-enero 1993). Informe técnico. División de Epidemiología, Servicio Seccional de Salud. Junio 1993:10.

5. Benavides ML, Realpe LO. Evaluación del tiempo en que Aedes aegypii coloniza un criadero doméstico en Neiva. Resúmenes, Seminario-Taller, Asociacion Colombiana de Ciencias Biológicas, Capítulo del Huila. El Juncal, junio 1993:4.

6. Forattini OP. Entomología médica. Culicini: Culex, Aedes e Psorophora. Sao Paulo: Universidade de Sao Paulo; 1965:505. 